\title{
Long term intravenous prostaglandin (epoprostenol or iloprost) for treatment of severe pulmonary hypertension
}

\author{
T Higenbottam, A Y Butt, A McMahon, R Westerbeck, L Sharples
}

\begin{abstract}
Objective-To investigate the relation between the severity of pulmonary hypertension and the outcome of medical treatment.

Methods-98 patients with primary pulmonary hypertension-nine $(6 \%)$ with systemic disease and pulmonary hypertension and 39 (27\%) with thromboembolic pulmonary hypertensionreceived medical treatment and were followed between 1982 and 1995. They were given long term intravenous prostaglandin treatment (either epoprostenol $(n=61)$ or iloprost $(n=13)$ ) or conventional treatment with oral anticoagulants ( $n=24)$ with or without calcium channel blockers. Event-free survival was measured to death or transplant surgery, or pulmonary thromboendarterectomy.

Results-Prognosis (hazard ratio) was affected by: New York Heart Association grade, 1.52 (95\% confidence interval 1.11 to 2.09); mixed venous oxygen saturation $\left(\mathrm{Svo}_{2} \%\right), 0.97$ (0.95 to 0.98); cardiac index, $0.72(0.49$ to 1.06$)$; mean right atrial pressure, 1.04 (1.01 to 1.07$)$; and pulmonary vascular resistance, $1.02(1.00$ to 1.04$)$. The median event-free survival time of patients with $\mathrm{Svo}_{2}<60 \%$ was 239 days ( 0 to $502)$ on conventional treatment $(n=22)$ and 585 days ( 300 to 870 ) on prostaglandin treatment $(n=42)$. No difference was seen in patients with $\mathrm{Svo}_{2} \geqslant 60 \%$ between conventional treatment and prostaglandin treatment, survival being 1275 days (732 to 1818; $(n=48)$ ) and 986 days (541 to 1431 ; $\mathbf{n}=30)$ ), respectively. Capacity for pulmonary vasodilatation did not predict
\end{abstract} outcome of treatment.

Conclusions-Continuous intravenous prostaglandins were more effective than anticoagulants, with or without calcium channel blockers, in prolonging survival in patients with right heart failure. In these patients a capacity to vasodilate did not predict outcome from medical treatment.

(Heart 1998;80:151-155)

Keywords: prostacyclin; iloprost; pulmonary hypertension; event-free survival

Despite its rarity ${ }^{1}$ and the effectiveness of treatment with continuous intravenous prostaglandin, ${ }^{2}$ many patients with primary pulmonary hypertension remain untreated. This reflects concern about the cost of the $\mathrm{drug}^{3}$ and uncertainty over the selection criteria for those patients who might benefit most.

To determine which patients are most likely to benefit from prostaglandin treatment we embarked on a retrospective analysis of the factors affecting survival of a large population of patients with severe pulmonary hypertension, considering in particular their treatment and the severity of their disease.

\section{Methods}

Between October 1982 and November 1995, 146 patients with pulmonary hypertension were admitted to Papworth Hospital for investigation and treatment. Diagnosis was made by right heart catheter, recording mean right atrial pressure (mRAP), mean pulmonary artery pressure (mPAP), mean systemic arterial pressure (mSAP), and cardiac output/cardiac index. Pulmonary artery blood samples were taken for measurement of mixed venous oxygen gas tension $\left(\mathrm{SvO}_{2} \%\right)$. The capacity to dilate the pulmonary circulations was also assessed during the catheter study. An accumulative dose response to intravenous prostacyclin $^{4}$ was undertaken, giving incremental steps of $2 \mathrm{ng} / \mathrm{kg} / \mathrm{min}$, until there was either an increase in cardiac index of more than $30 \%$ or a fall in mSAP of $20 \%$. A $30 \%$ increase in cardiac index was taken to denote a capacity to vasodilate.

Further investigations included chest $x$ ray, echocardiography ( $M$ mode and Doppler), full lung function tests, and a ventilation/perfusion (V/Q) lung scan, together with full immunological screening for connective tissue disease to determine the presence of secondary pulmonary hypertension. Pulmonary thromboembolic disease was diagnosed when there were two or more segmental or subsegmental perfusion defects on the V/Q scan which were normally ventilated. ${ }^{5}$

A decision to treat patients with a long term intravenous infusion of prostaglandins was made on the basis of the severity of their disease. The main indication was exercise tolerance, measured by a 12 minute walking distance $^{6}$ of less than $500 \mathrm{~m}$; very few of the patients were New York Heart Association (NYHA) class I. A request for funding was then made to the patient's local health authority. In a proportion of cases this was accepted and the infusion of epoprostenol (prostacyclin) or iloprost was started. 
Table 1 Patient characteristics and haemodynamics

\begin{tabular}{|c|c|c|c|}
\hline Variable & $\begin{array}{l}\text { Prostaglandin } \\
\text { treatment }\end{array}$ & $\begin{array}{l}\text { Conventional } \\
\text { treatment }\end{array}$ & p value \\
\hline Age (years) & 34.0 & 43.0 & 0.22 \\
\hline \multicolumn{4}{|l|}{ Sex } \\
\hline Male & 26 & 28 & \multirow[t]{2}{*}{0.77} \\
\hline Female & 48 & 44 & \\
\hline \multicolumn{4}{|l|}{ NYHA } \\
\hline Grade I and II & 3 & 32 & \multirow[t]{2}{*}{$<0.001$} \\
\hline Grade III and IV & 69 & 39 & \\
\hline \multicolumn{4}{|l|}{ Diagnosis } \\
\hline $\mathrm{PPH}$ & 59 & 49 & \multirow{2}{*}{0.16} \\
\hline Secondary & 15 & 23 & \\
\hline \multicolumn{4}{|l|}{ Vasodilator response } \\
\hline None & 24 & 26 & \multirow[t]{2}{*}{0.73} \\
\hline Acute & 37 & 33 & \\
\hline \multicolumn{4}{|l|}{$\mathrm{SvO}_{2}$} \\
\hline$<60 \%$ & 42 & 22 & \multirow[t]{2}{*}{0.002} \\
\hline$\geqslant 60 \%$ & 30 & 48 & \\
\hline \multicolumn{4}{|l|}{ Right heart catheter } \\
\hline \multicolumn{4}{|l|}{ Before vasodilator } \\
\hline mRAP $(\mathrm{mm} \mathrm{Hg})^{\star}$ & 12.0 & 8.0 & 0.005 \\
\hline $\mathrm{mPAP}(\mathrm{mm} \mathrm{Hg}) \dagger$ & $66.9(16.6)$ & $58.0(16.7)$ & 0.001 \\
\hline $\mathrm{SvO}_{2}(\%) \dagger$ & $56.5(9.0)$ & $63.0(11.2)$ & $<0.001$ \\
\hline $\mathrm{CO}(1 / \mathrm{min})^{\star}$ & 2.8 & 3.6 & $<0.001$ \\
\hline $\mathrm{CI}\left(1 / \mathrm{min} / \mathrm{m}^{2}\right)^{\star}$ & 1.7 & 2.1 & $<0.001$ \\
\hline PVR (Wood units) ${ }^{\star}$ & 18.7 & 14.6 & $<0.001$ \\
\hline \multicolumn{4}{|l|}{ After vasodilator } \\
\hline $\operatorname{mPAP}(\mathrm{mm} \mathrm{Hg}) \dagger$ & $64.6(17.6)$ & $55.6(16.4)$ & 0.004 \\
\hline $\mathrm{SvO}_{2}(\%) \dagger$ & $64.6(8.7)$ & $71.4(9.7)$ & $<0.001$ \\
\hline $\operatorname{CO}(1 / \mathrm{min})^{\star}$ & 3.6 & 4.8 & $<0.001$ \\
\hline $\operatorname{PVR}(w u)^{\star}$ & 15.5 & 10.0 & $<0.001$ \\
\hline
\end{tabular}

${ }^{\star}$ Median values; Mann-Whitney test used for comparison.

†Mean (SD) values; unpaired $t$ test used for comparison.

$\mathrm{A} \chi^{2}$ test was used to compare proportions.

$\mathrm{CO}$, cardiac output; CI, cardiac index; mPAP, mean pulmonary arterial pressure; mRAP, mean right atrial pressure; NYHA, New York Heart Association grade; $\mathrm{PPH}$, primary pulmonary hypertension; PVR, pulmonary vascular resistance; secondary, pulmonary hypertension secondary to systemic disease or thromboembolism; $\mathrm{SvO}_{2}$, mixed venous oxygen saturation.

\section{PROSTAGLANDIN TREATMENT}

Delivery into the subclavian vein through a subcutaneous tunnel has already been described in detail. ${ }^{7}$ In the prostaglandin treated patients, the choice of epoprostenol or iloprost, an analogue of prostacyclin which became available in 1989, was made according to the patient's preference. Iloprost is pharmacologically equivalent to epoprostenol but is more stable and easier to use at home. ${ }^{8}{ }^{9}$ Ease of use was the patients' principal reason for choosing iloprost. For the purposes of this study we have combined patients using either drug into one group.

CONVENTIONAL TREATMENT

In those patients for whom agreement to fund prostaglandin treatment was not received, anticoagulants were continued. Patients who had shown a capacity for vasodilatation at right heart catheter study were also treated with oral calcium channel blockers in a dose to maintain a systolic systemic blood pressure of $100 \mathrm{~mm} \mathrm{Hg} .{ }^{10}$ Calcium channel blockers included nifedipine, diltiazem, and amlodipine. This constituted conventional treatment and no patient on prostaglandins continued conventional treatment. This contrasts with the work of Barst et $a l^{2}$ where the patients all continued conventional treatment.

\section{OUTCOME MEASURES}

The primary outcome measure for this study was time until death or surgery. The surgical treatment was either heart-lung transplantation or pulmonary endarterectomy. A deterioration in the patients' clinical status determined whether a patient was treated surgically.
All patients were seen at three monthly intervals and the 12 minute walking distance measured. When exercise distance fell to the level seen at the time of diagnosis, patients were assigned to surgical treatment. They were offered heart-lung transplantation ${ }^{11}$ or thromboendarterectomy where there was central pulmonary thrombotic obstruction. ${ }^{12}$ Time from this point to surgery depended on the availability of donors or access to specialist surgical facilities for thromboendarterectomy.

STATISTICAL ANALYSIS

Values are given as mean $(95 \%$ confidence interval). The Mann-Whitney or unpaired $t$ test was used to compare haemodynamic variables and basic patient characteristics between the groups. The $\chi^{2}$ test was used to compare the sex distribution between the two main treatment groups. Cox's proportional hazards regression analysis was used to calculate the hazards ratios in the univariate and multivariate analysis, in order to determine independent predictors of death or surgery. Significance levels were calculated using the likelihood ratio test. For the survival analysis, the KaplanMeier method was used to calculate the median survival times and the survival curves, using the log rank test to compare them. A value of less than $5 \%$ was taken to be significant.

Two subgroup analyses were undertaken. (1) Patients were divided according to $\mathrm{SvO}_{2}$. The prognosis of patients with severe pulmonary hypertension depends on the presence of right ventricular failure. ${ }^{13}{ }^{14}$ The degree of reduction in cardiac index, rise in $\mathrm{mRAP}$, and increase in pulmonary vascular resistance (PVR) provide a guide to survival. The $\mathrm{SvO}_{2} \%$ also characterises the patients' survival chances, ${ }^{14}{ }^{15}$ so we divided the patients into two groups according to this measurement: those with a poor prognosis have an $\mathrm{SvO}_{2}$ of $<60 \%$, while those with a better prognosis have an $\mathrm{SvO}_{2}$ of $\geqslant 60 \% .{ }^{15}$ (2) In the other subgroup analysis we divided the patients into those who did or did not have a capacity to vasodilate.

\section{Results}

Of the 146 patients, 98 had primary pulmonary hypertension $(67 \%)$, nine $(6 \%)$ had severe pulmonary hypertension associated with systemic disease (systemic lupus erythematosus in two, systemic sclerosis in four, and sarcoidosis in three), while 39 (27\%) had thromboembolic pulmonary hypertension. At the end of the study, in November 1995, 20 patients (14\%) had needed heart-lung transplantation, two ( $1 \%)$ had undergone a pulmonary thromboendarterectomy, and $72(49 \%)$ had died, leaving $52(36 \%)$ alive. Overall median survival to death or surgery was 695 days (95\% confidence interval 546 to 844 days). For those 22 patients who underwent surgery, the median survival time was 695 days (426 to 964). For those 72 who died, the median survival time was 345 days (240 to 456 ).

The characteristics and haemodynamic measurements of the patients are shown in table 1, divided by treatment group into those 
Table 2 Univariate analysis of the prognostic factors

\begin{tabular}{|c|c|c|}
\hline Variable & Hazard ratio $(95 \% \mathrm{CI})$ & p value \\
\hline Diagnosis, PPH $v$ secondary $\mathrm{PH}$ & $1.22(0.97$ to 1.53$)$ & 0.096 \\
\hline Treatment, PT $v$ CT & $0.98(0.79$ to 1.21$)$ & 0.816 \\
\hline Vasodilator response & $0.93(0.73$ to 1.18$)$ & 0.555 \\
\hline NYHA (class III and IV) & $1.52(1.11$ to 2.09$)$ & 0.009 \\
\hline Sex (male) & $1.07(0.87$ to 1.32$)$ & 0.494 \\
\hline Age (/year increase) & $1.00(0.98$ to 1.02$)$ & 0.900 \\
\hline $\mathrm{SvO}_{2}(/ \%$ increase $)$ & $0.97(0.95$ to 0.98$)$ & $<0.001$ \\
\hline $\mathrm{CO}$ (/litre/min increase $)$ & $0.77(0.62$ to 0.97$)$ & 0.016 \\
\hline $\mathrm{CI}\left(/\right.$ litre $\left./ \mathrm{min} / \mathrm{m}^{2}\right)$ & $0.72(0.49$ to 1.06$)$ & 0.082 \\
\hline mRAP (/mm Hg increase) & $1.04(1.01$ to 1.07$)$ & 0.018 \\
\hline PVR (Wood units) & $1.02(1.00$ to 1.04$)$ & 0.047 \\
\hline $\mathrm{mPAP}(/ \mathrm{mm} \mathrm{Hg}$ increase) & $1.00(0.99$ to 1.01$)$ & 0.763 \\
\hline
\end{tabular}

Hazard ratio analysis using a univariate analysis. The $\mathrm{p}$ value is derived from the likelihood ratio test.

PT, prostaglandin treatment; CT, conventional treatment; CI, cardiac index; CO, cardiac output; mPAP, mean pulmonary artery pressure; mRAP mean right atrial pressure; NYHA, New York Heart Association grade; PPH, primary pulmonary hypertension; PVR, pulmonary vascular resistance; secondary $\mathrm{PH}$, pulmonary hypertension secondary to systemic disease and thromboembolic disease; $\mathrm{SvO}_{2}$, mixed venous oxygen saturation.

receiving prostaglandin treatment or conventional treatment.

Factors such as NYHA grade and $\mathrm{SvO}_{2} \%$ are known to be strong indicators of the severity of a patient's condition, together with mPAP, PVR, and cardiac index. ${ }^{13-15}$ As anticipated, there was a tendency for the most severely affected patients to be given prostaglandins. Only three $(9 \%)$ of the 35 patients in NYHA grade I and II were given prostaglandins, compared with $69(64 \%)$ of the 108 patients in class III and IV. Similarly the $\mathrm{SvO}_{2} \%$ of the prostaglandin treatment group was $6.5 \%$ lower than in the conventional treatment group. Strong evidence of a difference was also shown by the higher mPAP in the prostaglandin group $(8.9 \mathrm{~mm} \mathrm{Hg}$ higher than in the conventional treatment group) and the higher median PVR (4.1 units higher). All these differences were significant.

Univariate analysis was performed on each of the factors in table 1 to investigate the hazards associated with each. These results are shown in table 2.

Several variables were found to be strongly predictive of early death or surgery. The strongest predictor was $\mathrm{SvO}_{2} \%$, with a significant hazard ratio $(p<0.001)$. This ratio indicates that the lower the value of $\mathrm{SvO}_{2}$, the higher the risk of death or surgery. The NYHA grade was also a strong predictor, with a significant hazard ratio $(\mathrm{p}=0.009)$, indicating that grades III or IV were associated with a greater risk than grades I or II. The other significant predictors were cardiac index, mRAP, and PVR. As previously reported, mPAP was not a significant predictor. As the data involve many hidden interrelations, the only accurate way to assess the importance of the prognostic factors-especially of treatment-is through multivariate analysis. In the univariate analysis, the effect of the patient's condition is not taken into account, therefore strongly affecting the assessment of the treatment as the more severely affected patients were given prostaglandins. In the multivariate analysis, the patient's condition can be accounted for, so the true value of the treatment can be assessed.

The multivariate analysis incorporated a model with a selection of the factors investi-
Table 3 Multivariate analysis of the prognostic factors

\begin{tabular}{lll}
\hline Variable & $\begin{array}{l}\text { Hazard ratio } \\
\text { (95\% CI) }\end{array}$ & p value \\
\hline Treatment, PT $v$ CT & $0.78(0.60$ to 1.01$)$ & 0.0649 \\
NYHA (class III and IV) & $1.40(0.98$ to 2.00$)$ & 0.0620 \\
Svo $_{2}$ (/\% increase) & $0.97(0.95$ to 0.99$)$ & 0.0077 \\
PVR (/wu increase) $^{1.01(0.99 \text { to } 1.04)}$ & 0.3938 \\
\hline
\end{tabular}

Multivariate analysis of the prognostic factors where $\mathrm{p}$ value is from the likelihood ratio test. The treatment effect does not quite achieve significance overall for the population.

PT, prostaglandin treatment; CT, conventional treatment; $\mathrm{SvO}_{2}$ (mixed venous oxygen saturation) was a significant predictor; NYHA (New York Heart Association) grade just failed to reach significance; PVR (pulmonary vascular resistance) was not a significant predictor.

gated in the univariate analysis. The primary objective was to find a true estimate of the effect of treatment, while allowing for the other influential factors. Therefore the model included the measures of the patients' overall condition, which were $\mathrm{SvO}_{2} \%$ and NYHA grade, together with PVR (table 3).

When assessing the effectiveness of prostaglandin treatment, the importance of allowing for measures of the patients' condition is clearly shown in table 3 . The hazard ratio associated with prostaglandin treatment fell from 0.95 in the univariate analysis to 0.78 in the multivariate, only just missing significance $(p=0.06)$. The value obtained from the multivariate analysis is therefore likely to be close to the true hazard ratio for treatment, but there could still be hidden interrelations in the data. The hazard ratio associated with NYHA grade changed little, while that for $\mathrm{SvO}_{2} \%$ did not change, indicating that these were likely to be the true values for these factors. It should be noted that the hazard ratio for PVR did not change significantly either.

To evaluate the effect of treatment further, we compared the survival of the two treatment groups within different strata. The first objective was to compare survival in the two treatment groups within the lower and higher $\mathrm{SvO}_{2} \%$ strata, as this is such a strong indicator of the severity of the patients' condition. The second objective was to compare survival in the groups with an acute vasodilator response and in those without. This would offer a possible method of predicting the performance of each treatment.

Of the 64 patients with an $\mathrm{SvO}_{2} \%$ of less than $60 \%, 22$ were given conventional treatment and 42 were given prostaglandin treatment. The median survival for patients with conventional treatment was 239 days ( 0 to 502 ), while in those treated with prostaglandins it was 585 days (300 to 870 ). There was a significant difference to the Kaplan-Meier survival plot $(\mathrm{p}=0.02)$. Of the 78 patients with an $\mathrm{SvO}_{2} \%$ greater than $60 \%, 48$ received conventional treatment and 30 were given prostaglandin treatment. The median survival time of the conventional treatment group was 1275 days (732 to 1818), while for the prostaglandin treatment group it was 986 days (541 to 1431). There was no difference in the survival curves $(p=0.5)$. Therefore for the more severely affected patients prostaglandin treatment significantly improved survival, by almost a 
year, in contrast to the less severely affected patients, in whom there was no effect on survival.

Of the 50 patients who showed no vasodilatation on diagnostic catheterisation, 26 received conventional treatment and 24 received prostaglandin treatment. The median survival for the conventional treatment group was 899 days (197 to 1601 ) and it was similar for the prostaglandin group (797 days (47 to 1547)). There was no difference in the survival curves $(p=0.1)$. Of the 70 acute responders, 33 had conventional treatment and 37 prostaglandin treatment. For the conventional treatment group, median survival time was not available as only $10(30.3 \%)$ reached the end point of death or surgery. For the prostaglandin treatment group, median survival time was 776 days (620 to 932). There was no significant difference between the two Kaplan-Meier survival curves $(p=0.07)$. There was no evidence that the capacity to vasodilate predicted the performance of either conventional treatment or prostaglandin treatment.

\section{Discussion}

Strong indicators of prognosis in earlier studies of the natural history of primary pulmonary hypertension ${ }^{13-15}$ and pulmonary hypertension from congenital heart disease ${ }^{15}$ are NYHA grade, cardiac index, mRAP, and PVR, together with the $\mathrm{SvO}_{2} \%{ }^{14}{ }^{15}$ By stratifying the patients according to their $\mathrm{SvO}_{2}$-above or below $60 \%$-we observed that in the most severely affected patients, prostaglandin treatment enhanced their event-free survival by almost a year. An $\mathrm{Svo}_{2} \%$ value below $60 \%$ therefore predicts those patients with severe pulmonary hypertension who will benefit from long term intravenous infusion of epoprostenol or iloprost. The presence or absence of a capacity for acute pulmonary vasodilatation does not predict the performance of prostaglandin treatment.

As this study was not a randomised trial, various problems naturally occur. The statistical techniques used in the analysis are based on the assumption that the sample of patients in the study is a random one from one whole population, which is not the case. Treatments were not allocated randomly, and were intentionally given to the more severely affected patients. This could have lessened the impact of treatment on survival. We tried to overcome this throughout the analysis by including measures of the patients' condition as covariates. There was evidence that the hazard ratio associated with prostaglandin was $78 \%$ of that with conventional treatment, although this just missed significance at the $5 \%$ level $(\mathrm{p}=0.06)$. It is possible that the hazard ratio associated with prostaglandin treatment would be lower than $78 \%$ if more measures of clinical condition had been included, such as exercise tolerance and arterial blood gases. Rather than increasing the complexity of the study, we chose instead to analyse the results according to severity of right ventricular failure, categorising the groups according to $\mathrm{SvO}_{2} \%$. The survival analysis of the two treatment groups within the lower $\mathrm{SvO}_{2} \%$ band showed that prostaglandin treatment was effective. The ideal way to show this benefit of prostaglandin treatment would be a randomised controlled trial. However, this study has two major attributes-a large sample size and a long duration of follow up-which give strong indications of the efficacy of long term intravenous epoprostenol or iloprost treatment.

The identification of patients with right ventricular failure offers a practical guide for selecting those patients most likely to respond to prostaglandin treatment. These patients can be recognised by high mRAP values, a low cardiac index, an NYHA grade of III and IV, or a low $\mathrm{SvO}_{2} \%$. However, a capacity for pulmonary vasodilatation does not predict success in this group. For patients with no evidence of right ventricular failure and in whom the pulmonary circulation can be dilated during diagnostic catheterisation, anticoagulants and oral calcium channel blocker treatment offers a means of improving event-free survival. ${ }^{10}$

As the medical treatment of primary pulmonary hypertension and pulmonary hypertension from the different secondary causes appears to be similar, we combined patients with thromboembolic pulmonary hypertension, pulmonary hypertension secondary to systemic disease, and primary pulmonary hypertension. For example, in patients with pulmonary hypertension from systemic diseases such as systemic sclerosis, prostaglandin treatment appears to be as effective as in primary pulmonary hypertension. ${ }^{9}$ Treatment with oral vasodilators and anticoagulants also appears effective in the treatment of thromboembolic pulmonary hypertension. ${ }^{16}$

We cannot determine the optimum time for heart-lung or lung transplantation from this study. However, survival after transplant surgery is about $60 \%$ at 18 months in most centres ${ }^{17}$ which is comparable with the results of prostaglandin treatment in our study. The relative costs and effects on the quality of life of the two treatments need to be considered in detail. From the limited data, models have been developed to describe relative cost and benefit. From these models, it appears that the relative cost per index of quality of life (quality adjusted life year) is similar for the two approaches. ${ }^{18} 19$ A case can therefore be made for delaying transplant surgery until prostaglandin treatment fails.

We wish to thank Mr John Wallwork, director of the Papworth Hospital Cardiothoracic Transplant Unit, for continued and productive collaboration.

1 Abenheim I, Morides Y, Brenot F, et al. Appetitesuppressant drugs and risk of primary pulmonary hype tension. N Engl f Med 1996;335:609-16.

2 Barst R, Rubin L, Long W, et al. A comparison of continuous intravenous epoprostenol (prostacyclin) with conventional therapy for primary pulmonary hypertension. $N$ Engl f Med 1996;334:296-301.

3 Higenbottam TW. Prostacyclin. Bandolier 1995;14:3-4.

4 Jones DK, Higenbottam TW, Wallwork J. Treatment of primary pulmonary hypertension with intravenous epoprostenol (prostacyclin). Br Heart $\mathcal{F}$ 1987;57:270-8.

5 Stein PD, Hull RD, Saltzman HA et al. Strategy for diagnosis of patients with suspected pulmonary emboli. Chest 1993;103:1553-9.

6 McGavin CR, Gupta SP, McHardy GJR. Twelve minute walking test for assessing disability in chronic bronchitis. $B M F 1976 ; \mathrm{i}: 822-3$. 
7 Higenbottam TW, Wheeldon D, Wells F, et al. Long-term treatment of primary pulmonary hypertension with continuous intravenous epoprostenol (prostacyclin). Lancet 1984;i:1046-7.

8 De La Mata J, Gomez-Sanchez MA, Aranzana M, et al. Long-term iloprost infusion therapy for severe pulmonary hypertension in patients with connective tissue diseases. Arthritis Rheum 1994;37:1528-33.

9 Butt AY, Dinh-Xaun T, Takao M, et al. Treatment of pulmonary hypertension with the continuous infusion of a prostacyclin analogue iloprost. Heart 1998;79:175-9.

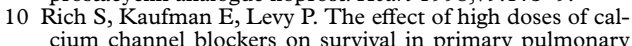
hypertension. N Engl f Med 1992;327:76-81.

11 Dawkins KD, Jamieson SW, Hunt SA, et al. Long-term results, hemodynamics, and complications after combined heart and lung transplantation. Circulation 1985;71:919heart
26.

12 Moser KM, Fedullo PF, Auger WR, et al. Chronic thromboembolic pulmonary hypertension: clinical picture thromboembolic pulmonary hypertension: clinical pical treatment. Eur Resp f 1992;5:334-43.

13 D'Alonzo GE, Barst RJ, Ayres SM. Survival in patients with primary pulmonary hypertension. Ann Intern Med 1991 115:343-9.
14 Fuster V, Steele PM, Edwards WD. Primary pulmonary hypertension: natural history and importance of thrombosis. Circulation 1984;70:580-7.

15 Higenbottam TW, Spiegelhalter D, Scott J, et al. Prostacycin (epoprostenol) and heart-lung transplantation as treatments of severe pulmonary hypertension. Br Heart $\mathcal{F}$ 1993;70:366-70.

16 Hopkins WE, Ochoa LL, Richardson GW, et al. Comparison of the hemodynamics and survival of adults with severe primary pulmonary hypertension or Eisenmenger's syndrome. F Heart Lung Transplant 1996;15:100-5.

17 Brown G. Pharmacological treatment of primary and secondary pulmonary hypertension. Pharmacology 1991; 11:137-56.

18 Hosenpud JD, Novick RJ, Bennett LE, et al. The registry of the International Society for Heart and Lung Transplantation: thirteenth official report-1996. F Heart Lung Transplant 1996;15:655-74.

19 Higenbottam TW, Ward SE, Brennan A, et al. Prostacyclin in the treatment of primary pulmonary hypertension. Sheffield: of Leicester, Nottingham and Sheffield, 1997. (Guidance note for purchasers: $97 / 02$.) 